and the creation of a dedicated role or team may be necessary.

Unfortunately, lots of the fluids that surgical instruments come into contact with on a daily basis are also their key enemies. Blood, pus, surgical debris, dish or laundry soap, bleach, iodine-type and chlorhexidine solutions are all primary causes of staining and pitting of instruments.

The washing process should begin within 20 minutes after surgery, even if sterilization will take place much later. Only solutions that specifically state 'for surgical instruments' on the label should be used. After cleaning all items should be visually inspected, including all surfaces, cannulations, joints, lumens and ratchets.

\section{STERILIZATION OF INSTRUMENTS}

All surgical instruments need to be sterilized ready for surgical procedures. They must be clean and free from blood before being placed in the autoclave, as the temperature $\left(121-132^{\circ} \mathrm{C}\right)$ will cause chemical reactions that can make the stain permanent.

Instruments should be sterilized with their ratchets open to allow better steam penetration and prevent the box joints from cracking. If packed within a tray, then it should be perforated to allow better steam penetration and more effective drying. For efficiency, heavy instruments should be placed at the bottom and lighter, more delicate instruments on top. If sterilizing in paper or plastic pouches, they should not be stacked during sterilization and, if possible, pouches should be sterilized on their side. It is important that the stated maximum load for the autoclave is not exceeded.

\section{MAINTENANCE}

A maintenance plan should include servicing, restoration and sharpening of instruments on a regular basis and in a proactive approach. All members of the team should have the knowledge to be able to identify faults to remove those items from general use.

\section{KEY LEARNING OBJECTIVES}

Analyse current methods of cleaning to ensure the correct methods are being used

- Understand how regular care and maintenance prolong the lifespan of instruments

- Identify common faults in instruments and equipment and how to resolve them

\section{MULTIPLE CHOICE QUESTIONS}

1. What product should be used to clean surgical instruments after a procedure?
(A) Chlorhexidine solutions
(B) Washing-up liquid
(C) Soap (pH7-8)
(D) Cold soak solution

2. How should instruments with box joints be sterilized?

(A) With box joint open

(B) With box joint fully closed

(C) With box joint closed on first click

(D) Does not matter

3. When should instruments be checked for damage?
(A) By the surgeon at the beginning of the procedure
(B) Prior to washing the instrument
(C) After washing but prior to sterilization
(D) After sterilization

\section{Surgery into the beyond: how to mend a broken heart}

\section{Alison Young}

It is estimated that approximately $10 \%$ of dogs presented to primary care veterinary practices have heart disease, and chronic valvular heart disease (CHVD) is the most common heart disease of dogs in many parts of the world, accounting for approximately $75 \%$ of canine cases of heart disease cases seen by veterinary practices in North America.

\section{MITRAL VALVE DISEASE}

Myxomatous mitral valve disease is usually a degenerative condition. The valve edges become thickened and allow leakage of blood back into the atrium. The chordae tendineae, sometimes referred to as the 'heart strings' since they resemble small pieces of string, attach to the papillary muscles and hold the atrioventricular valves in place while the heart is pumping. The chordae tendineae can become elongated and even rupture, causing the valve edges to be pulled even further away from each other and allowing even more blood to regurgitate back into the atrium.

\section{MEDICAL MANAGEMENT}

Many dogs with early disease do not, and may never, require treatment for their disease. Dogs that develop signs of failure are very likely to ultimately succumb to their disease.

\section{SURGICAL TREATMENT - MITRAL VALVE REPAIR}

The aim of surgery is to reduce the volume of leakage through the valve, which will also enable the heart to 


\section{Surgical/Theatre}

remodel, and allow the left atrium to shrink back down to a more normal size. To achieve this valve repair is performed. This consists of replacing any ruptured or stretched chords and placement of a purse-string suture around the valve annulus to draw it back down to a smaller size.

\section{NURSING ROLES}

A scrub nurse running the instrument trolley must ensure the correct equipment and instruments are available and pre-empt the surgeon's needs. As a surgical assistant they provide retraction of the atrial wall for the sutures to be placed.

The nurses work closely with the anaesthesia team to help transfer the patient back to the intensive care unit at the end of the procedure. Patients require continuous monitoring of temperature, pulse, respiration, blood pressure, arterial blood gas analysis, chest drain output and urine output.

\section{PROGNOSIS}

The ability to perform open heart surgery for dogs with mitral valve disease has brought hope to a lot of dog owners across the world. What was a certain countdown to congestive heart failure and the awful death that it could cause, can now be delayed. This procedure aims for the patient to be medication-free by 3 months postoperatively.

\section{KEY LEARNING OBJECTIVES}

- Identify patients for whom surgery may now be an option

- Understand the aims of the surgery and the postoperative needs of the patient

- Evaluate and develop the nurse's role within the team

\section{MULTIPLE CHOICE QUESTIONS}

1. What is the estimated percentage of dogs presented to their veterinary surgeon with heart disease have CVHD?
(A) $75 \%$
(C) $50 \%$
(B) $10 \%$
(D) $87 \%$

2. What is the current estimated median survival time for a dog after they have experienced an episode of congestive heart failure (ACVIM classification stage $\mathrm{C})$ ?
(A) 2-4 months
(B) 12-18 months
(C) 9-12 months
(D) 2-3 years

3. What is the main function of the bypass machine during this procedure?
(A) Heart and lung function
(B) Provide anaesthesia
(C) Filter excess fluid
(D) Kidney function 